\title{
ESTIMATES OF CALORIC MEASURE AND THE INITIAL-DIRICHLET PROBLEM FOR THE HEAT EQUATION IN LIPSCHITZ CYLINDERS
}

BY

EUGENE FABES ${ }^{1}$ AND SANDRO SALSA ${ }^{2}$

\begin{abstract}
In this paper the authors prove unique solvability of the initial-Dirichlet problem for the heat equation in a cylindrical domain with Lipschitz base, lateral data in $L^{p}, p \geqslant 2$, and zero initial values. A Poisson kernel for this problem is shown to exist with the property that its $L^{2}$-averages over parabolic rectangles are equivalent to $L^{1}$-averages over the same sets.
\end{abstract}

Introduction. Let $D$ denote a bounded Lipschitz domain in $R^{n}$ and $D_{+}$the cylinder $D \times(0, \infty)$. Given a continuous function $g$ on $\partial D_{+}$and compactly supported there, there exists a unique classical solution, $u$, of the heat equation

$$
L u(x, t) \equiv \sum_{i=1}^{n} D_{x_{i}}^{2} u(x, t)-D_{t} u(x, t)=0 \text { in } D_{+}
$$

with initial-boundary data $g$. When we fix a point $(x, t) \in D_{+}$the mapping $g \rightarrow u(x, t)$ is a continuous nonnegative linear functional on $C\left(\partial D_{+}\right)$and hence there exists a unique Borel measure, $\omega^{x, t}$, on $\partial D_{+}$so that $u(x, t)=\int_{\partial D_{+}} g d \omega^{x, t}$. The measure $\omega^{x, t} \equiv \omega_{D_{+}}^{x, t}$ is called the caloric measure (associated with $D_{+}$) evaluated at $(x, t)$. Because of Harnack's inequality [7] for nonnegative solutions of the heat equation, for $T$ fixed and positive all the measures $\left\{\omega^{x, T}: x \in D\right\}$ are mutually absolutely continuous.

In this work we examine the relations between the surface measure and the caloric measure, $\omega^{x, T}$, restricted to the lateral boundary, $\partial D \times(0, T)$, of $D \times(0, T)$. Our main result states that caloric measure and surface measure on $\partial D \times(0, T)$ are mutually absolutely continuous. Moreover, if $d Q$ denotes surface measure on $\partial D$ then $d \omega^{x, T}(Q, \Delta)=K(x, T ; Q, s) d Q d s$ and for any parabolic surface cylinder $\Delta$ of $\partial D \times(0, T)$ (see notations below)

$$
\left[\frac{1}{|\Delta|} \int_{\Delta} K^{2}(x, T ; Q, s) d Q d s\right]^{1 / 2} \leqslant \frac{C}{|\Delta|} \int_{\Delta} K(x, T ; Q, s) d Q d s
$$

where $|\Delta|=\int_{\Delta} d Q d s$ and $C$ is a constant depending only on dimension, the Lipschitz character of $D$ and $(x, T) . C$ can be taken independent of the points $(x, T)$ provided these points are allowed to vary over a fixed compact subset of $D_{+}$.

Received by the editors September 9, 1981 and, in revised form, September 22, 1982.

1980 Mathematics Subject Classification. Primary 35K05, 35K20; Secondary 31B10, 31B25.

${ }^{1}$ This author was supported in part by the N.S.F.

${ }^{2}$ This author was supported in part by the G.N.A.F.A. 
The above result leads to the solvability of the initial-Dirichlet problem for the heat equation with given lateral data in $L^{p}, p \geqslant 2$. More precisely, if $g \in$ $L^{p}(\partial D \times(0, T))$ with $p \geqslant 2$, then there exists a unique solution of $L u=0$ in $D \times(0, T)$ satisfying the zero initial condition and at almost every point, $(Q, s)$, of $\partial D \times(0, T)$ converging to $g(Q, s)$ through parabolic cones with vertex at $(Q, s)$ (see Theorem 3.2).

We point out that for general domains in $R^{n+1}$ whose boundary is composed of a base and a lateral part, $S$, which is described locally by the graph, $x_{n}=$ $f\left(x_{1}, \ldots, x_{n-1}, t\right)$, with $f$ Lipschitz continuous in the space variable and Hölder continuous of order $\frac{1}{2}$ in time, caloric measure and $n$-dimensional Hausdorff measure on $S$ may be mutually singular. For details and further references the reader should see [10].

Before continuing, the authors wish to thank Professor Carlos E. Kenig for the many helpful discussions related to this paper. These discussions and his work with David Jerison provided us with the basic ideas and tools needed for the results.

Our paper is divided into three sections. $\$ 1$ proves the absolute continuity of $\omega^{x, T}$ with respect to surface measure on $\partial D \times(0, T)$ and establishes the $L^{2}$-integrability of the Poisson kernel,

$$
K(x, T ; Q, s)=\frac{d \omega^{x, T}(Q, s)}{d(d Q \times d s)}
$$

The main tool of this section is the identity in Lemma 1.1, which is a parabolic version of the identity 2.1 in [7] for the Poisson kernel of Laplace's equation.

$\$ 2$ studies the boundary behavior of the Poisson integral,

$$
u(x, t)=\int_{\partial D_{+}} K(x, t ; Q, s) f(Q, s) d Q d s \equiv \int_{S} f d \omega^{x, t},
$$

for $f \in L^{2}(\partial D \times(0, T))$. The primary result is the pointwise domination of the maximal function

$$
u^{*}\left(Q_{0}, s_{0}\right)=\sup _{\Gamma_{Q_{0}, s_{0}}|u|}
$$

( $\Gamma_{Q_{0}, s_{0}}$ is a parabolic cone with vertex at $Q_{0}, s_{0}$ and contained in $D \times(0, T)$ ) by a constant times the (parabolic) Hardy-Littlewood maximal function $M\left(f^{2}\right)\left(Q_{0}, s_{0}\right)^{1 / 2}$ where

$$
\begin{aligned}
M(g)\left(Q_{0}, s_{0}\right)=\sup _{r>0}\left\{\frac{1}{\left|\Delta_{r}\right|} \int_{\Delta_{r}}|g| d Q d s: \Delta_{r}\right. \text { is parabolic surface cylinder } \\
\left.\quad \text { of } \partial D \times(0, T) \text { with radius } r \text { and center } Q_{0}, s_{0}\right\} .
\end{aligned}
$$

In $\S 3$ we combine the results of $\S 2$ with the fundamental work of Muckenhoupt in [9] to conclude the mutual absolute continuity of caloric measure, $\omega^{x, T}$, and surface measure on $\partial D \times(0, T)$. Another consequence is the construction of the solution to the initial-Dirichlet problem for the heat equation with zero initial values and lateral data equal to a given $g \in L^{p}(\partial D \times(0, T)), p \geqslant 2$. 
Much of the notation we will use is either standard and needs no elaboration or will be explained at the time it is introduced. For now we wish to give the basic geometric definitions and objects repeatedly used in this work.

Our primary domains will be the cylinders $D_{+}=D \times(0, \infty)$ and $D_{T}=D \times(0, T)$ for $T<\infty$ and their corresponding lateral boundaries $S=\partial D \times(0, \infty)$ and $S_{T}=$ $\partial D \times(0, T)$. Here $D$ is a bounded region in $R^{n}$. The letters $x$ and $y$ will denote points in $R^{n}$ and will generally belong to $D . x^{\prime}$ and $y^{\prime}$ will denote points in $R^{n-1}$ and we will often write $x=\left(x^{\prime}, x_{n}\right)$ when we wish to distinguish the $n$th coordinate of $x$. Letters $P$ and $Q$ will denote points on $\partial D$, the boundary of $D$, and $t$ and $s$ will be used for time variables.

Definition. We call a bounded domain $D \subset R^{n}$ a Lipschitz domain if for each point $Q \in \partial D$ there exist a ball, $B \equiv B(r, Q)$, with center $Q$ and radius $r$, and a coordinate system of $R^{n}$ such that in these coordinates

$$
B \cap D=B \cap\left\{\left(x^{\prime}, x_{n}\right): x_{n}>\varphi\left(x^{\prime}\right) \text { and }\|\nabla \varphi\|_{L^{\infty}\left(R^{n-1}\right)} \leqslant m<\infty\right\}
$$

and $B \cap \partial D=B \cap\left\{\left(x^{\prime}, \varphi\left(x^{\prime}\right)\right): x^{\prime} \in R^{n-1}\right\}$.

We will assume the radius of the ball, $B$, and the constant $m$ in the above definition are chosen independent of $Q \in \partial D$. This radius, $r_{0}$, and the number $m$ define what we will call the local Lipschitz character of $D$. If in a given estimate the constant depends only on the dimension, $n, m, r_{0}$, and the number of coordinate balls of radius $r_{0}$ needed to cover $\partial D$, then we will say the "constant depends only on the Lipschitz character of $D$ ".

Finally we set

$$
\begin{aligned}
& \psi_{r}(Q, t)=\left\{(y, s): y \in D,|y-Q|<r,|t-s|<r^{2}, 0<s<T\right\}, \\
& \Delta_{r}(Q, t)=\left\{(P, s): P \in \partial D,|P-Q|<r,|t-s|<r^{2}, 0<s<T\right\} .
\end{aligned}
$$

$\Delta_{r}(Q, t)$ will be called the (parabolic) surface cylinder of $S_{T}$ with radius $r$ and center $Q, t$.

Throughout the rest of this paper $D$ will denote a bounded Lipschitz domain in $R^{n}$. If the functions, $\varphi$, whose graphs describe $\partial D$ locally are $C^{\infty}\left(R^{n-1}\right)$, then $D$ is called a smooth domain.

1. The $L^{2}$-integrability of the Poisson kernel. In this section we turn our attention to the absolute continuity of caloric measure with respect to surface measure on $S=\partial D \times(0, \infty)$ and to the square integrability of the Radon-Nikodym derivative, which is the Poisson kernel.

We set

$$
\Gamma(x, t)=(4 \pi t)^{-n / 2} \exp \left(-|x|^{2} / 4 t\right),
$$

the fundamental solution in all space for the heat equation, and, for the bounded Lipschitz domain, $D$, we denote by $G(x, y, t)$ the Green's function associated with $D$ and the heat operator. Specifically for $x, y \in D$ and $t>0, G(x, y, t)=$ $\Gamma(x-y, t)-g(x, y, t)$ where for $x$ fixed $g(x, \cdot, \cdot)$ is the unique solution of the problem

$$
L(g(x, \cdot, \cdot))=0, \quad g(x, y, 0)=0, \quad g(x, Q, t)=\Gamma(x-Q, t) \quad(Q \in \partial D) .
$$


For smooth domains, $D$, if $N_{Q}$ denotes the inner normal to $\partial D$ at $Q$, the function, $\left(\partial G / \partial N_{Q}\right)(x, Q, t-s)$, is the Poisson kernel for $L$ in $D_{+}$, i.e., the potential,

$$
v(x, t)=\int_{0}^{t} \int_{\partial D} \frac{\partial G}{\partial N_{Q}}(x, Q, t-s) f(Q, s) d Q d s,
$$

is the solution to the problem $L v=0$ in $D_{+}, v(x, 0)=0,\left.v\right|_{S}=f$. With obvious abuse of previous notation for $K$ we set

$$
K(x, t ; Q, s) \equiv K(x, Q, t-s) \equiv \frac{\partial G}{\partial N_{Q}}(x, Q, t-s) .
$$

LEMma 1.1. Fix $x_{0} \in D$ and $t_{0}>0$. Assume $V(y, s)$ is a $C^{\infty}$ vector field defined on $R^{n+1}$ with values in $R^{n}$ such that $V\left(x_{0}, t_{0}\right)=0$. For smooth domains, $D$, the following identity holds:

$$
\begin{aligned}
\int_{0}^{t_{0}} \int_{\partial D}\left(V(Q, s) \cdot N_{Q}\right) K\left(x_{0}, Q, t_{0}-s\right) K\left(x_{0}, Q, s\right) d Q d s \\
=-\int_{0}^{t_{0}} \int_{D} L\left(\frac{\partial g}{\partial V(y, s)}\left(x_{0}, y, s\right)\right) G\left(x_{0}, y, t_{0}-s\right) d y d s \\
\quad-\int_{0}^{t_{0}} \int_{\partial D} \frac{\partial \Gamma}{\partial V(Q, s)}\left(x_{0}, Q, s\right) K\left(x_{0}, Q, t_{0}-s\right) d Q d s
\end{aligned}
$$

where

$$
\frac{\partial \Gamma}{\partial V(Q, s)}\left(x_{0}, Q, s\right)=\frac{\partial}{\partial V(Q, s)}\left(\Gamma\left(x_{0}-\cdot, s\right)\right)(Q)
$$

and $V \cdot N$ is the standard inner product on $R^{n}$.

Proof. If $Q \in \partial D$ we can write $V(Q, s)=T(Q, s)+c(Q, s) N_{Q}$ where for each $s$, $T(Q, s)$ is a tangential vector field to $\partial D$. Since $G(x, Q, s)=0$ on $S$ we have

$$
\frac{\partial G}{\partial T(Q, s)}(x, Q, s) \equiv \frac{\partial}{\partial T(Q, s)}(G(x, \cdot, s))(Q)=0 \text {. }
$$

Hence

$$
\frac{\partial G}{\partial V(Q, s)}(x, Q, s)=c(Q, s) \frac{\partial G}{\partial N_{Q}}(x, Q, s)=c(Q, s) K(x, Q, s) .
$$

It follows that

$$
\begin{aligned}
& \int_{0}^{t} \int_{\partial D} \frac{\partial G}{\partial V(Q, s)}\left(x_{0}, Q, s\right) K(x, Q, t-s) d Q d s \\
& \quad=\int_{0}^{t} \int_{\partial D} c(Q, s) K\left(x_{0}, Q, s\right) K(x, Q, t-s) d Q d s
\end{aligned}
$$

for $x_{0}, x \in D$ and $t \in(0, T]$. 
Recall that $G\left(x_{0}, y, s\right)=\Gamma\left(x_{0}-y, s\right)-g\left(x_{0}, y, s\right)$. Hence the left side of (1.2) equals

$$
\begin{aligned}
& -\int_{0}^{t} \int_{\partial D} \frac{\partial \Gamma}{\partial V(Q, s)}\left(x_{0}, Q, s\right) K(x, Q, t-s) d Q d s \\
& -\int_{0}^{t} \int_{\partial D} \frac{\partial g}{\partial V(Q, s)}\left(x_{0}, Q, s\right) K(x, Q, t-s) d Q d s .
\end{aligned}
$$

Finally,

$$
\begin{aligned}
& \int_{0}^{t} \int_{\partial D} \frac{\partial g}{\partial V(Q, s)}\left(x_{0}, Q, s\right) K(x, Q, t-s) d Q d s \\
& \quad=\int_{0}^{t} \int_{D} L\left(\frac{\partial g}{\partial V(y, s)}\left(x_{0}, y, s\right)\right) G(x, y, t-s) d y d s+\frac{\partial g}{\partial V(x, t)}\left(x_{0}, x, t\right) .
\end{aligned}
$$

We now substitute in (1.2) to obtain

$$
\begin{aligned}
\int_{0}^{t} \int_{\partial D} c(Q, s) K\left(x_{0}, Q, s\right) K(x, Q, t-s) d Q d s \\
=-\int_{0}^{t} \int_{D} L\left(\frac{\partial g}{\partial V(y, s)}\left(x_{0}, y, s\right)\right) G(x, y, t-s) d y d s \\
\quad-\int_{0}^{t} \int_{\partial D} \frac{\partial \Gamma}{\partial V(Q, s)}\left(x_{0}, Q, s\right) K(x, Q, t-s) d Q d s-\frac{\partial g}{\partial V(x, t)}\left(x_{0}, x, t\right) .
\end{aligned}
$$

Since $\partial g\left(x_{0}, x_{0}, t_{0}\right) / \partial V\left(x_{0}, t_{0}\right)=0$ equation (1.1) follows after observing that $c(Q, s)=V(Q, s) \cdot N_{Q}$.

Lemma 1.2. Assume $D$ is smooth and let $m$ and $r_{0}$ determine the local Lipschitz character of $D$. For $Q_{0} \in \partial D, s_{0}>0$, and $0<r \leqslant r_{0} / 4$, set

$$
\left(Q_{r}, t_{r}\right)=\left(Q_{0}^{\prime}, Q_{0 n}+3 r, s_{0}+5 r^{2}\right) .
$$

There exists a constant $c$ depending only on the dimension $n$ and the local Lipschitz character of $D$ such that $\int_{\Delta_{r}\left(Q_{0}, s_{0}\right)} K\left(Q_{r}, Q, t_{r}-s\right) K\left(Q_{r}, Q, s\right) d Q d s \leqslant c r^{-n-1}$.

Proof. Pick $\theta=\theta(y, s) \in C_{0}^{\infty}\left(R^{n+1}\right)$ satisfying $0 \leqslant \theta \leqslant 1, \theta \equiv 0$ outside the set $\{(y, s):|y|<2,|s|<2\}$ and $\theta \equiv 1$ on $\{(y, s):|y| \leqslant 1,|s| \leqslant 1\}$. Consider the vector field

$$
V_{r}(y, s)=\theta\left(\frac{y-Q_{0}}{r}, \frac{s-s_{0}}{r^{2}}\right) e_{n} \quad\left(e_{n}=(0,-, 0,1) \in R^{n}\right)
$$

and observe that

$$
V_{r}(Q, s) \cdot N_{Q} \geqslant \theta\left(\frac{Q-Q_{0}}{r}, \frac{s-s_{0}}{r^{2}}\right) \frac{1}{\sqrt{1+m^{2}}} .
$$


We now apply Lemma 1.1 with $V$ replaced by $V_{r}, x_{0}$ by $Q_{r}, t_{0}$ by $t_{r}=s_{0}+5 r^{2}$ and we obtain the inequality,

$$
\begin{aligned}
\frac{1}{\sqrt{1+m^{2}}} \int_{\Delta_{r}\left(Q_{0}, s_{0}\right)} K\left(Q_{r}, Q, t_{r}-s\right) K\left(Q_{r}, Q, s\right) d Q d s \\
\leqslant\left|\int_{0}^{t_{r}} \int_{D} L\left(\frac{\partial g}{\partial V_{r}(y, s)}\left(Q_{r}, y, s\right)\right) G\left(Q_{r}, y, t_{r}-s\right) d y d s\right| \\
\quad+\left|\int_{0}^{t_{r}} \int_{D} \frac{\partial \Gamma}{\partial V_{r}(Q, s)}\left(Q_{r}, Q, s\right) K\left(Q_{r}, Q, t_{r}-s\right) d Q d s\right|=\mathrm{I}+\text { II. }
\end{aligned}
$$

We have

$$
|\mathrm{II}| \leqslant C \cdot \sup \left\{\left|D_{y_{n}} \Gamma\left(Q_{r}, Q, s\right)\right|:(Q, s) \in \Delta_{2 r}\left(Q_{0}, s_{0}\right)\right\} \leqslant c \cdot r^{-n-1} .
$$

To get an estimate for $I$, notice that

$$
\begin{aligned}
L\left(\frac{\partial g\left(Q_{r}, y, s\right)}{\partial V_{r}(y, s)}\right) & =\left(D_{s}-\Delta_{y}\right) \theta_{r} \cdot D_{y_{n}} g \\
& =\left(D_{s} \theta_{r}\right) D_{y_{n}} g-\left(\Delta_{y} \theta_{r}\right) \cdot D_{y_{n}} g-2 \sum_{i=1}^{n}\left(D_{y_{i}} \theta_{r}\right) \cdot D_{y_{i} y_{n}}^{2} g .
\end{aligned}
$$

By our choice of $\theta_{r}$ we have

$$
\left|D_{y} \theta_{r}\right|<c \cdot r^{-1}, \quad\left|D_{t} \theta_{r}\right|<c \cdot r^{-2}, \quad\left|D_{y}^{2} \theta_{r}\right|<c \cdot r^{-2} .
$$

Performing an integration by parts in the terms involving $D_{y_{i} y_{n}}^{2} g$ and using the above estimates we get

$$
\begin{aligned}
|\mathrm{I}| & \leqslant c \cdot\left\{\frac{1}{r} \int_{\psi_{2 r}\left(Q_{0}, s_{0}\right)}\left|\nabla_{y} G\left(Q_{s}, y, t_{r}-s\right)\right|\left|\nabla_{y} g\left(Q_{r}, y, s\right)\right| d y d s\right. \\
& \left.\quad+\frac{1}{r^{2}} \int_{\psi_{2 r}\left(Q_{0}, s_{0}\right)} G\left(Q_{r}, y, t_{r}-s\right)\left|\nabla_{y} g\left(Q_{r}, y, s\right)\right| d y d s\right\} \\
& =c \cdot(A+B) .
\end{aligned}
$$

We show how to estimate $A$, the way to estimate $B$ being analogous. By Schwarz' inequality

$$
\begin{aligned}
|A| \leqslant & \frac{1}{r}\left(\int_{\psi_{2 r}}\left|\nabla_{y} \Gamma\left(Q_{r}, y, t_{r}-s\right)\right|^{2} d y d s\right)^{1 / 2}\left(\int_{\psi_{2 r}}\left|\nabla_{y} g\left(Q_{r}, y, s\right)\right|^{2}\right)^{1 / 2} \\
& +\frac{1}{r}\left(\int_{\psi_{2 r}}\left|\nabla_{y} g\left(Q_{r}, y, s\right)\right|^{2} d y d s\right)^{1 / 2}\left(\int_{\psi_{2 r}}\left|\nabla_{y} g\left(Q_{r}, y, t_{r}-s\right)\right|^{2}\right)^{1 / 2} .
\end{aligned}
$$

Since

$$
\left(\int_{\psi_{2 r}}\left|\nabla_{y} \Gamma\left(Q_{r}, y, t_{r}-s\right)\right|^{2} d y d s\right)^{1 / 2} \leqslant c r^{-n / 2}
$$


we have to show that

$$
\begin{gathered}
\left(\int_{\psi_{2 r}}\left|\nabla_{y} g\left(Q_{r}, y, s\right)\right|^{2} d y d s\right)^{1 / 2} \leqslant c r^{-n / 2}, \\
\left(\int_{\psi_{2 r}}\left|\nabla_{y} g\left(Q_{r}, y, t_{r}-s\right)\right|^{2} d y d s\right)^{1 / 2} \leqslant c \cdot r^{-n / 2} .
\end{gathered}
$$

Since the procedure for estimating both integrals is the same, we will give the details only for the first one.

We consider

$$
\Re_{r} \equiv\left\{(y, s):\left(\left|y-Q_{r}\right|^{2}+\left|s-t_{r}\right|\right)^{1 / 2}<\alpha r\right\}
$$

with $\alpha$ chosen in a way such that $\psi_{2 r}\left(Q_{0}, s_{0}\right) \cap \Re_{r}=\varnothing$. Now let $h=h(y, s) \in$ $C^{\infty}\left(\mathbf{R}^{n+1}\right)$ with $h \equiv 1$ outside $\mathcal{K}_{r}$ and $h \equiv 0$ in $\mathcal{K}_{r / 2}$. Denote by $w=w(y, s)$ the solution of the problem

$$
L w=L(h \Gamma), \quad w=0 \text { on } S \cup\{D x\{0\}\} ; \text { here } \Gamma=\Gamma\left(Q_{r}-\cdot, \cdot\right) .
$$

We have $g\left(Q_{r}, y, s\right)=h(y, s) \Gamma\left(Q_{r}-y, s\right)-w(y, s)$, and what we wish to estimate now are the integrals

$$
I_{1}=\int_{\psi_{2 r}}\left|\nabla_{y}(h \Gamma)\right|^{2} \quad \text { and } \quad I_{2}=\int_{\psi_{2 r}}\left|\nabla_{y} w\right|^{2} .
$$

In $\psi_{2 r} h \Gamma=\Gamma$, therefore $\left|\nabla_{y}(h \Gamma)\right|<c \cdot r^{-n-1}$ and $\left|I_{1}\right| \leqslant c \cdot r^{-2 n-2} \cdot r^{n+2}=c \cdot r^{-n}$, the desired bound.

To estimate $I_{2}$, consider a smooth function $\varphi(y, s)$ such that $0 \leqslant \varphi \leqslant 1, \varphi(y, s)$ $\equiv 1$ in $\psi_{2 r}, \varphi(y, s) \equiv 0$ outside $\psi_{(2+\gamma) r}$ with $\gamma$ chosen in a way such that $\psi_{(2+\gamma) r} \cap \Re_{r}$ $=\varnothing$. Then, from $L w=L(h \Gamma)$ we get

$$
\int_{\psi(2+\gamma) r} \varphi^{2} w L w=\int_{\psi_{(2+\gamma) r}} \varphi^{2} w L(h \Gamma) .
$$

Using integration by parts we have

$$
\begin{aligned}
\int_{\psi_{(2+\gamma) r}} \varphi^{2} w L w & =\int_{\psi_{(2+\gamma) r}} \varphi^{2}\left(\frac{1}{2} D_{t} w^{2}-w \Delta_{y} w\right) \\
& =\int_{\psi_{(2+\gamma) r}} \varphi^{2}\left|\nabla_{y} w\right|^{2}-\int_{\psi_{(2+\gamma) r}} \varphi w^{2} D_{t} \varphi+\int_{\psi_{(2+\gamma) r}} 2 w \varphi \nabla_{y} \varphi \cdot \nabla_{y} w .
\end{aligned}
$$

Therefore

$$
\begin{aligned}
\int_{\psi_{(2+\gamma) r}} \varphi^{2}\left|\nabla_{y} w\right|^{2} \leqslant & \int_{\psi_{(2+\gamma) r}} \varphi^{2} w|L(h \Gamma)|+\int_{\psi_{(2+\gamma) r}} \varphi\left|D_{t} \varphi\right| w^{2} \\
& +\int_{\psi_{(2+\gamma) r}} 2 w \varphi\left|\nabla_{y} \varphi\right|\left|\nabla_{y} w\right| .
\end{aligned}
$$


Using the estimates $\left|\nabla_{y} \varphi\right|<c \cdot r^{-1}$ and $\left|D_{t} \varphi\right|<c \cdot r^{-2}$ we get

$$
\begin{aligned}
\int_{\psi(2+\gamma) r} \varphi^{2}\left|\nabla_{y} w\right|^{2} \leqslant & \int_{\psi(2-\gamma) r}|w L(h \Gamma)|+\frac{c}{r^{2}} \int_{\psi(2 \cdot \gamma) r} w^{2} \\
& +\frac{1}{2} \int_{\psi(2+\gamma) r} \varphi^{2}\left|\nabla_{y} w\right|^{2}+\frac{2}{r^{2}} \int_{\psi(2+\gamma) r} w^{2} .
\end{aligned}
$$

The latter inequality implies

$$
\int_{\psi_{2 r}}\left|\nabla_{y} w\right|^{2} \leqslant \frac{c}{r^{2}} \int_{\psi_{2 r}} w^{2}+\int_{\psi_{(2+\gamma) r}}|w L(h \Gamma)| .
$$

Since $|w| \leqslant 2 \Gamma \leqslant c r^{-n-2}$ we conclude that

$$
I_{2}=\int_{\psi_{2 r}}\left|\nabla_{y} w\right|^{2} \leqslant \frac{c}{r^{2}} r^{n+2} \frac{1}{r^{2 n}}+\frac{c}{r^{n}} r^{n+2} \frac{1}{r^{n+2}}=c r^{-n}
$$

which is the desired bound for $I_{2}$.

Remark. Suppose $0 \in D$. Then the arguments of Lemma 1.2 can be used to show that

$$
\int_{0}^{T} \int_{\partial D} K(0, Q, T-s) \cdot K(0, Q, s) d Q d s \leqslant c
$$

where the constant depends only on the dimension, the Lipschitz character of $D$ and $\max (1, T)$. To see this, choose $\theta=\theta(y) \in C_{0}^{\infty}\left(\mathbf{R}^{n}\right)$ satisfying $0 \leqslant \theta \leqslant 1, \theta \equiv 0$ outside the set $\{y,|y|<2\}, \theta \equiv 1$ on $\{y,|y| \leqslant 1\}$ and consider the vector field $V_{Q}(y, s)=V_{Q}(y)=\theta\left((y-Q) / \alpha r_{0}\right) \cdot e_{n}$ where $\alpha$ is chosen in a way such that $V_{Q}(0, s)=0$ for each $Q \in \partial D$. Now cover the boundary $\partial D$ by a finite number of surface balls $S_{k} \equiv B\left(\alpha r_{0}, Q_{k}\right) \cap \partial D, k=1, \ldots, N, N$ depending only on the Lipschitz character of $D$ and repeat verbatim the arguments in the proof of Lemma 1.2 with $V_{Q_{k}}, 0, T$ instead of $V_{r}, Q_{r}, t_{r}$, respectively.

We are now ready to prove the main theorems of this section. These results assume $D$ is a bounded Lipschitz domain in $R^{n}$ containing the origin, 0 .

THEOREM 1.3. Let $\omega^{0, T}$ be the caloric measure in $D_{+}$evaluated at $(0, T)$ and $d Q \times d t$ the $n$-dimensional Hausdorff or surface measure on $S_{T}$. Then $\omega^{0, T} \ll d Q \times d t$ with density in $L^{2}\left(S_{T}\right)$.

THEOREM 1.4. The limit

$$
\frac{\partial}{\partial N_{Q_{0}}} G\left(0, Q_{0}, T-s_{0}\right)=\lim _{r \rightarrow 0} \nabla_{y} G\left(0, Q_{0}+r N_{Q_{0}}, T-s_{0}\right) \cdot N_{Q_{0}}
$$

exists for a.e. (with respect to $d Q \times d t)\left(Q_{0}, s_{0}\right) \in S$. Furthermore, for each Borel set $E \subset S$ we have

$$
\omega^{0, T}(E)=\int_{E} \frac{\partial}{\partial N_{Q}} G(0, Q, T-s) d Q d s .
$$

Proof of Theorem 1.3. Assume first that $D$ is smooth. Since $K(x, Q, t)=$ $\left(\partial / \partial N_{Q}\right) G(x, Q, t)$, as a function of $(x, t), Q$ fixed on $\partial D$, is a nonnegative solution 
of the heat equation, the Harnack principle [8] implies that

$$
K(0, Q, s) \leqslant c K(0, Q, 3 T-s) \text { if } s \in[0, T] .
$$

Thus by the above inequality and the Remark following Lemma 1.2 we can write

$$
\begin{aligned}
\int_{0}^{T} \int_{\partial D} K^{2}(0, Q, T-s) d Q d s & =\int_{0}^{T} \int_{\partial D} K^{2}(0, Q, s) d Q d s \\
& \leqslant c \int_{0}^{3 T} \int_{\partial D} K(0, Q, s) K(0, Q, 3 T-s) d Q d s \leqslant c,
\end{aligned}
$$

where the constant depends only on the dimension, the Lipschitz character of $D$, and $\max (T, 1)$.

Suppose now that $D$ is Lipschitz and $B$ is a ball with center $Q \in \partial D$ for which

$$
B \cap \partial D=\left\{\left(y^{\prime}, \varphi\left(y^{\prime}\right)\right): y^{\prime} \in R^{n-1},\|\nabla \varphi\|_{L^{x}} \leqslant m\right\} .
$$

We can find a sequence of smooth domains, $D^{j}$, and a sequence of smooth functions, $\varphi_{j}$, such that (a) $\varphi_{j} \rightarrow \varphi$ uniformly, (b) $\nabla \varphi_{j} \rightarrow \nabla \varphi$ a.e. in $R^{n-1}$, (c) $\left\|\nabla \varphi_{j}\right\|_{L^{x}} \leqslant 2 m$, and (d) $B \cap\left\{\left(y^{\prime}, \varphi_{j}\left(y^{\prime}\right)\right)\right\} \subset \partial D^{j}$.

Denote by $\omega_{j}$ the caloric measure associated with $D_{+}^{j}$ and suppose $f$ is a continuous function on $R^{n+1}$ whose support is compactly contained in $B_{+}=B \times$ $(0, \infty)$. From Lemma 1.2 and the preceding argument in the smooth case

$$
\begin{aligned}
\omega_{j}^{(0, T)}(f) & =\int_{0}^{T} \int_{\partial D^{j}} f\left(Q_{j}, s\right) K_{j}\left(0, Q_{j}, T-s\right) d Q_{j} d s \\
& \leqslant c\|f\|_{L^{2}\left(S_{T}^{\prime}\right)} \quad\left(S_{T}^{j}=\partial D^{j} \times(0, T)\right)
\end{aligned}
$$

with $c$ independent of $j$.

Since $\omega_{j}^{(0, T)}(f) \rightarrow \omega^{0, T}(f)$ and $\|f\|_{L^{2}\left(S_{T}^{\prime}\right)} \rightarrow\|f\|_{L^{2}\left(S_{T}\right)}$ as $j \rightarrow \infty$ we have $\omega^{0, T}(f) \leqslant$ $c\|f\|_{L^{2}\left(S_{T}\right)}$. By using a partition of unity of $\partial D$ corresponding to a finite covering of coordinate balls $B$ we obtain the estimate

$$
\omega^{0, T}(f) \leqslant c\|f\|_{L^{2}\left(S_{T}\right)} .
$$

An application of the Riesz Representation Theorem concludes the proof of (1.3).

REMARK. It is clear from the argument in Theorem 1.3 that

$$
K(x, t ; Q, s)=\frac{d \omega^{x, t}}{d Q \times d s}
$$

is zero for $s>t$.

Proof of Theorem 1.4. Fix $\left(Q_{0}, s_{0}\right) \in S_{T}$ and let $B_{0}$ denote the ball of radius $r_{0}$ and center $Q_{0}$ for which, in the coordinate system $y=\left(y^{\prime}, y_{n}\right), B_{0} \cap D=B_{0} \cap\{y$ : $\left.y_{n}>\varphi\left(y^{\prime}\right)\right\}$. Let $\Gamma\left(Q_{0}, s_{0}\right)$ denote the parabolic cone with vertex $\left(Q_{0}, s_{0}\right)$ given by $\left\{(y, s): c>y_{n}-Q_{0 n}>c^{\prime}\left|y^{\prime}-Q_{0}^{\prime}\right|+c^{\prime \prime}\left|s-s_{0}\right|^{1 / 2}, 0<s<T\right\}$. Since $D$ is Lipschitz we can find $c, c^{\prime}, c^{\prime \prime}$ such that $\Gamma\left(Q_{0}, s_{0}\right) \subset D_{T}$. Set $d=d\left(y, s ; Q_{0}, s_{0}\right)$ $=\sqrt{\left|y-Q_{0}\right|^{2}+\left|s-s_{0}\right|}$.

From Schauder estimates and Harnack's inequality [8],

$$
\left|\nabla_{y} G(0, y, T-s)\right| \leqslant c \cdot d^{-1} G\left(0, Q_{d}, T-s_{0}+2 d^{2}\right)
$$


where $Q_{d}=\left(Q_{0}^{\prime}, Q_{0 n}+d\right)$. From Lemma 2.2 of [10] we have

$$
d^{-n} G\left(0, Q_{d}, T-s_{0}+2 d^{2}\right) \leqslant c \omega^{(0, T)}\left(\Delta_{d}\left(Q_{0}, s_{0}-2 d^{2}\right)\right) .
$$

The last two inequalities imply

$$
\begin{aligned}
\left|\nabla_{y} G(0, y, T-s)\right| & \leqslant c d^{-n-1} \omega^{(0, T)}\left(\Delta_{2 d}\left(Q_{0}, s_{0}\right)\right) \\
& \leqslant c \sup _{r>0} r^{-n-1} \int_{\Delta_{r}\left(Q_{0}, s_{0}\right)} K(0, T ; Q, s) d Q d s \\
& =c M(K(0, T ; \cdot, \cdot))\left(Q_{0}, s_{0}\right) .
\end{aligned}
$$

Here

$$
K(0, T ; \cdot, \cdot)=d \omega^{(0, T)} / d(d \sigma \times d t)
$$

and $M$ is the Hardy-Littlewood maximal operator. By Theorem 1.3 and the Remark following it $M(K(0, T ; \cdot, \cdot))\left(Q_{0}, s_{0}\right)$ is finite for a.e. (with respect to $d Q \times d t$ ) point $\left(Q_{0}, s_{0}\right) \in S$; therefore $\left|\nabla_{y} g(0, y, T-s)\right|$ is nontangentially bounded a.e. $(d Q \times d t)$ on $S_{T}$. By Lemma 2.6 of [5] we get the first part of the theorem. The second part of the theorem can be proved by very closely following, with obvious changes, the argument in [2, Lemma 7(b)]. We omit the details.

REMARK. The argument for passing from smooth to Lipschitz domains given in the proof of Theorem 1.3 can be used directly to extend Lemma 1.2 to Lipschitz domains. As a consequence if we again abuse the notation for $K$ and set $K(x, Q, t)$ $=\left(\partial / \partial N_{Q}\right) G(x, Q, t)$ then

$$
\int_{\Delta_{r}\left(Q_{0}, s_{0}\right)} K\left(Q_{r}, Q, t_{r}-s\right) K\left(Q_{r}, Q, s\right) d Q d s \leqslant c r^{-n-1}
$$

with $C$ depending only on the Lipschitz character of $D$.

2. Boundary behavior of the Poisson integral. In this section we study the parabolic nontangential maximal function associated with the potential

$$
u(x, t)=\int_{S} f(Q, s) d \omega^{x, t}(Q, s) .
$$

For $Q_{0} \in \partial D$ we let $B_{0}$ denote the ball of radius $r_{0}$ and center $Q_{0}$ for which there exists a coordinate system of $R^{n}$ so that relative to this system

$$
B_{0} \cap D=B_{0} \cap\left\{y=\left(y^{\prime}, y_{n}\right): y_{n}>\varphi\left(y^{\prime}\right)\right\} .
$$

Also in terms of these same coordinates we describe the (inner) nontangential parabolic cone with vertex $\left(Q_{0}, s_{0}\right)$ by

$$
\Gamma\left(Q_{0}, s_{0}\right)=\left\{(y, s): c>y_{n}-Q_{0 n}>c^{\prime}\left|y^{\prime}-Q_{0}^{\prime}\right|+c^{\prime \prime}\left|s-s_{0}\right|^{1 / 2}, 0<s<T\right\}
$$

where $c, c^{\prime}, c^{\prime \prime}$ are constants depending only on the local Lipschitz character of $D$ and chosen so that $\Gamma\left(Q_{0}, s_{0}\right) \subset D_{+}$.

If $u$ is a function defined on $D_{T}$ we set $u^{*}\left(Q_{0}, s_{0}\right)=\sup _{\Gamma\left(Q_{0}, s_{0}\right)}|u(y, s)|$ and we call $u^{*}$ the parabolic nontangential maximal function of $u$ at $\left(Q_{0}, s_{0}\right)$. Finally, as in 
the proof of Theorem 1.3, we let

$$
M(g)\left(Q_{0}, s_{0}\right)=\sup _{r>0} \frac{1}{r^{n+1}} \int_{\Delta_{r}}|g(Q, s)| d Q d s
$$

denote the (parabolic) Hardy-Littlewood maximal operator.

LEMMA 2.1. Suppose $f \in L^{2}(S)$ and $u(x, t)=\int_{S} f(Q, s) d \omega^{x, t}(Q, s)$. For each $T>0$ there exists a constant $c$ depending on the Lipschitz character of $D$ and $\max (T, 1)$ such that

$$
u^{*}\left(Q_{0}, s_{0}\right) \leqslant c M\left(f^{2}\right)\left(Q_{0}, s_{0}\right)^{1 / 2}
$$

for all $\left(Q_{0}, s_{0}\right) \in S_{T}=\partial D \times(0, T)$.

Proof. Clearly we can assume $f \geqslant 0$. By Harnack's inequality

$$
u^{*}\left(Q_{0}, s_{0}\right) \leqslant c \sup _{r>0} u\left(Q_{r}, t_{r}\right)
$$

where $\left(Q_{r}, t_{r}\right)=\left(Q_{0}^{\prime}, Q_{0 n}+3 r, s_{0}+5 r^{2}\right)$. As usual $c$ denotes a constant depending only on the dimension and on the local Lipschitz character of $D$.

Set $R_{0}=\Delta_{0}, R_{j}=\Delta_{j} \backslash \Delta_{j-1}$ where $\Delta_{j}=\Delta_{2^{j_{r}}}\left(Q_{0}, s_{0}\right)$. We fix $r$ with $r<r_{0}\left(r_{0}\right.$ defined in the Introduction) and choose $N$ so that $2^{N-1} r<r_{0} \leqslant 2^{N} r$. Then, we have

$$
u\left(Q_{r}, t_{r}\right) \leqslant \sum_{j=0}^{N} \int_{R_{j}} f(Q, s) d \omega^{\left(Q_{r}, t_{r}\right)}+\int_{\Delta_{r_{0}}^{c} \cap S_{T}} f(Q, s) d \omega^{\left(Q_{r}, t_{r}\right)} .
$$

From Lemma 1.2 of [5] we infer

$$
I=\int_{\Delta_{r_{0}}^{c} \cap S_{r}} f(Q, s) d \omega^{\left(Q_{r}, t_{r}\right)} \leqslant c \cdot \int_{\Delta_{r_{0}}^{c} \cap S_{T}} f(Q, s) d \omega^{\left(0, t_{r_{0}}\right)}(Q, s) \quad\left(t_{r_{0}}=s_{0}+5 r_{0}^{2}\right) .
$$

Theorem 1.1 and Schwarz' inequality give

$$
I \leqslant c\left(\int_{0}^{t_{r_{0}}} \int_{\partial D} f^{2}(Q, s) d Q d s\right)^{1 / 2} \leqslant c\left\{M\left(f^{2}\right)\left(Q_{0}, s_{0}\right)\right\}^{1 / 2} .
$$

Consider now $\int_{R_{j}} f(Q, s) d \omega^{\left(Q_{r}, t_{r}\right)}$. For $j=0$ we have

$$
\begin{aligned}
\int_{R_{0}} f(Q, s) d \omega^{\left(Q_{r}, t_{r}\right)}=\int_{\max \left(0, s_{0}-r^{2}\right)}^{s_{0}+5 r^{2}} \int_{\left|Q-Q_{0}\right|<r} f(Q, s) K\left(Q_{r}, Q, s_{0}+5 r^{2}-s\right) d Q d s \\
\quad \leqslant \int_{0}^{5 r^{2}} \int_{\left|Q-Q_{0}\right|<r} f\left(Q, s_{0}+5 r^{2}-s\right) K\left(Q_{r}, Q, s\right) d Q d s \\
\leqslant\left(\int_{\Delta_{3 r}\left(Q_{0}, s_{0}\right)} f^{2}(Q, s) d Q d s\right)^{1 / 2}\left(\int_{\Delta_{3 r}\left(Q_{0}, 0\right)} K^{2}\left(Q_{r}, Q, s\right) d Q d s\right)^{1 / 2} .
\end{aligned}
$$

Observe now that $K(x, Q, t)$ as a function of $(x, t)$ for $Q$ fixed on $\partial D$ is a nonnegative solution of $L u=0$. Since $s \in\left(0,9 r^{2}\right)$ we have $20 r^{2}-s>s$ and Harnack's inequality implies

$$
K\left(Q_{r}, Q, s\right) \leqslant c K\left(Q_{r}, Q, 20 r^{2}-s\right)
$$


with $c$ independent of $Q$ and $r$. By Lemma 1.2 and the Remark following Theorem 1.4

$$
\begin{aligned}
& \int_{\Delta_{3 r}\left(Q_{0}, 0\right)} K^{2}\left(Q_{r}, Q, s\right) d Q d s \\
& \quad \leqslant c \int_{\Delta_{3 r}\left(Q_{0}, 0\right)} K\left(Q_{r}, Q, s\right) K\left(Q_{r}, Q, 20 r^{2}-s\right) d Q d s \leqslant c r^{-n-1}
\end{aligned}
$$

Collecting the above estimates we obtain

$$
\int_{R_{0}} f(Q, s) d \omega^{\left(Q_{r}, t_{r}\right)} \leqslant c r^{(-n-1) / 2}\left(\int_{\Delta_{3 r}\left(Q_{0}, s_{0}\right)} f^{2}(Q, s) d Q d s\right)^{1 / 2} .
$$

For $j=1,2, \ldots, 6$ we proceed in the manner described above. Suppose now that $j>6$. The function $h(x, t)=\int_{R_{j}} f(Q, s) K(x, Q, t-s) d Q d s$ is a nonnegative solution of the heat equation vanishing in a neighborhood of $\Delta_{2^{j-2} r}\left(Q_{0}, s_{0}\right)$. The boundary Harnack principle in [6] implies

$$
h(x, t) \leqslant c h\left(Q_{2^{j} r}, t_{2^{j} r}\right)
$$

for $(x, t) \in \psi_{2^{j-2} r}\left(Q_{0}, s_{0}\right)$ with $c$ independent of $j$ and $r$.

We choose coordinates in $R^{n}$ so that $Q_{0}$ is the origin and

$$
\begin{array}{r}
\psi_{2^{j-2} r}\left(Q_{0}, s_{0}\right)=\left\{(x, t):|x|<2^{j-2} r,\left|t-s_{0}\right|<4^{j-2} r^{2}, x=\left(x^{\prime}, x_{n}\right)\right. \\
\text { with } \left.x_{n}>\varphi\left(x^{\prime}\right),\|\nabla \varphi\|_{L^{\infty}} \leqslant m, \varphi(0)=0\right\} .
\end{array}
$$

The function

$$
\tilde{u}(y, t)=\frac{h\left(2^{j-2} r y, 4^{j-2} r^{2} t+s_{0}\right)}{h\left(Q_{2^{j} r}, t_{2^{j} r}\right)}
$$

is defined on $\left\{(y, t):|y|<1,|t|<1, y=\left(y^{\prime}, y_{n}\right), y_{n}>\psi\left(y^{\prime}\right)\right\}$ where $\psi\left(y^{\prime}\right)=$ $\left(2^{j-2} r\right)^{-1} \varphi\left(2^{j-2} r y^{\prime}\right)$. $\tilde{u}$ also satisfies the heat equation and $|\tilde{u}| \leqslant c$ on this set. Moreover,

$$
\tilde{u}=0 \quad \text { on }\left\{(y, t):|y|<1,|t|<1, y=\left(y^{\prime}, \psi\left(y^{\prime}\right)\right)\right\} .
$$

Since $\|\nabla \psi\|_{L^{\infty}}=\|\nabla \varphi\|_{L^{\infty}}=m$ and $c$, the bound for $\tilde{u}$, depends only on $m, \tilde{u}$ is Hölder continuous at $(0,0)$ with a Hölder exponent and constant depending only on $m$. Hence

$$
h\left(Q_{r}, t_{r}\right)=h\left(Q_{2^{j_{r}}}, t_{2^{j_{r}}}\right) \tilde{u}\left(\frac{1}{2^{j-2}}, \frac{5}{4^{j-2}}\right) \leqslant \operatorname{ch}\left(Q_{2^{j_{r}}}, t_{2^{j_{r}}}\right) 2^{-j \alpha}
$$

with $c$ and $\alpha$ depending only on the local Lipschitz character of $D$.

Now set $c_{j}=2^{-j \alpha}$. Then

$$
h\left(Q_{r}, t_{r}\right) \leqslant c c_{j} \int_{\Delta_{2^{\prime} r}} f(Q, s) K\left(Q_{2^{j} r}, Q, 4^{j} r^{2}+s_{0}-s\right) d Q d s
$$


Again, a change of variables and Schwarz' inequality give

$$
\begin{aligned}
& \int_{\Delta_{2^{\prime} r}\left(Q_{0}, s_{0}\right)} f(Q, s) K\left(Q_{2^{\prime} r}, Q, 4^{j} r^{2}+s_{0}-s\right) d Q d s \\
& \leqslant c c_{j}\left(\int_{\Delta_{2^{j+1} r}\left(Q_{0}, s_{0}\right)} f^{2}(Q, s) d Q d s\right)^{1 / 2} \\
& \cdot\left(\int_{\Delta_{2^{\prime}+1_{r}\left(Q_{0}, 0\right)}} K^{2}\left(Q_{2^{j_{r}}}, Q, S\right) d Q d s\right)^{1 / 2} .
\end{aligned}
$$

The boundary Harnack principle [6] implies

$$
K\left(Q_{2^{j} r}, Q, s\right) \leqslant c K\left(Q_{2^{j} r}, Q, 5 \cdot 4^{j} r^{2}-s\right)
$$

for $s \in\left(0,4 \cdot 4^{j} r^{2}\right)$ with $c$ independent of $Q, r$ and $j$. The above estimate and Lemma 1.2 give

$$
\int_{\Delta_{2^{\prime+1}{ }_{r}}\left(Q_{0}, 0\right)} K^{2}\left(Q_{2^{\prime} r}, Q, s\right) d Q d s \leqslant c\left(2^{j} r\right)^{-n-1}
$$

Now (2.6), together with (2.5), gives

$$
\int_{R_{j}} f(Q, s) d \omega^{\left(Q_{r}, t_{r}\right)} \leqslant c c_{j}\left\{\left(2^{j+1} r\right)^{-n-1} \int_{\Delta_{2^{j+1} r}\left(Q_{0}, s_{0}\right)} f^{2}(Q, s) d Q d s\right\}^{1 / 2}
$$

with $\Sigma^{\infty} c_{j}<\infty$.

In conclusion we have, collecting all our estimates,

$$
u\left(Q_{r}, t_{r}\right) \leqslant c\left(\sum^{\infty} c_{j}\right)\left(M\left(f^{2}\right)\left(Q_{0}, s_{0}\right)\right)^{1 / 2}
$$

This concludes the proof of Lemma 2.1.

In the next lemma we assume $0 \in D$ and we set

$$
M_{\omega}(f)\left(Q_{0}, s_{0}\right)=\sup _{r>0} \frac{1}{\omega^{(0, T)}\left(\Delta_{r}\left(Q_{0}, s_{0}\right)\right)} \int_{\Delta_{r}\left(Q_{0}, s_{0}\right)}|f(Q, s)| d \omega^{(0, T)}(Q, s) .
$$

LEMma 2.2. Suppose $f \in L^{2}\left(S_{T}\right), f \geqslant 0$, and $u(x, t)=\int_{S_{T}} f(Q, s) d \omega^{(x, t)}(Q, s)$. Then, for $\left(Q_{0}, s_{0}\right) \in S_{T}$, we have

$$
c M_{\omega}(f)\left(Q_{0}, s_{0}\right) \leqslant u^{*}\left(Q_{0}, s_{0}\right) \leqslant C M_{\omega}(f)\left(Q_{0}, s_{0}\right)
$$

with $c$ and $C$ depending only on the dimension and on the Lipschitz character of $D$.

Proof. Clearly we may assume $f \geqslant 0$. The right inequality in Lemma 2.2 is an immediate consequence of $[5, \S 2]$. For the left one, we have

$$
\begin{aligned}
u^{*}\left(Q_{0}, s_{0}\right) & \geqslant u\left(Q_{r}, t_{r}\right)=\int_{S} f(Q, s) d \omega^{\left(Q_{r}, t_{r}\right)} \\
& \geqslant \int_{\Delta_{r}\left(Q_{0}, s_{0}\right)} f(Q, s) \tilde{K}\left(Q_{r}, Q, t_{r}, s\right) d \omega^{(0, T)} .
\end{aligned}
$$


In (2.8) we have set $\tilde{K}\left(Q_{r}, \cdot, t_{r}, \cdot\right)=d \omega^{\left(Q_{r}, t_{r}\right)} / d \omega^{(0, T)}$ which belongs to $L^{1}\left(S ; d \omega^{(0, T)}\right)$ by Harnack's inequality. By Lemma (1.4) of [5], we have

$$
1 \equiv \tilde{K}(0, Q, T, s) \leqslant c \tilde{K}\left(Q_{r}, Q, t_{r}-s\right) \omega^{(0, T)}\left(\Delta_{r}\left(Q_{0}, s_{0}\right)\right)
$$

and from (2.8) and (2.9) the desired inequality follows quite easily.

3. The initial-Dirichlet problem. In this section we prove our main results concerning the mutual absolute continuity of caloric and surface measure and the construction of a unique solution to the initial-Dirichlet problem in $D_{+}$with zero initial values and lateral boundary values equal to some given $L^{p}$ data, $p \geqslant 2$.

TheOREM 3.1. Assume $D$ is a bounded, Lipschitz domain in $R^{n}$ containing the origin. Let $\omega^{0, T}$ denote the caloric measure associated with $D_{+}$and evaluated at $(0, T)$. Set

$$
K(0, Q, T-s)=\frac{d \omega^{0, T}}{d(d Q \times d s)}(Q, s)
$$

where $d Q=$ surface measure on $\partial D$. Then there exists a constant $c$ depending only on $n$, the Lipschitz character of $D$ and $\max (T, 1)$ so that for any surface cylinder $\Delta$ of $S_{T}$ the following inequality holds:

$$
\left(\frac{1}{|\Delta|} \int_{\Delta} K^{2}(0, Q, T-s) d Q d s\right)^{1 / 2} \leqslant c \frac{1}{|\Delta|} \int_{\Delta} K(0, Q, T-s) d Q d s
$$

where $|\Delta|=\int_{\Delta} d Q d s$.

Consequently, $d Q \times d s$ and $\omega^{0, T}$ are mutually absolutely continuous on $S_{T}=\partial D \times$ $(0, T)$.

Proof. Lemmas 2.1 and 2.2 imply that $M_{\omega}(f)$ is a bounded operator from $L^{2}\left(S_{T}\right)$ into weak- $L^{2}\left(S_{T}\right)$. Now applying the results in [9] we conclude that $M_{\omega}(f)$ is bounded from $L^{2}\left(S_{T}\right)$ into $L^{2}\left(S_{T}\right)$ and that (3.1) holds. This in turn (see [1, Lemma 5]) implies that $d Q \times d s \ll \omega^{0, T}$ on $S_{T}$. The absolute continuity of $\omega^{0, T}$ with respect to surface measure was already proved in $\$ 2$.

THEOREM 3.2. Assume $f \in L^{p}\left(S_{T} ; d Q \times d t\right)$ with $p \geqslant 2$. Then there exists a unique solution $u$ of the heat equation in $D_{T}$ satisfying

(i) $\lim _{t \rightarrow 0^{+}} u(x, t)=0$ uniformly on compact subsets of $D$;

(ii) for almost every $(d Q \times d t)\left(Q_{0}, s_{0}\right) \in S_{T}$

$$
\lim _{\substack{(x, t) \in \Gamma\left(Q_{0}, s_{0}\right) \\(x, t) \rightarrow\left(Q_{0}, s_{0}\right)}} u(x, t)=f\left(Q_{0}, s_{0}\right)
$$

where $\Gamma\left(Q_{0}, s_{0}\right)$ is a parabolic cone contained in $D_{T}$ with vertex $Q_{0}$, $s_{0}$ (see the beginning of $\$ 2)$;

(iii) $u^{*}\left(Q_{0}, s_{0}\right) \in L^{p}\left(S_{T} ; d Q \times d t\right)$.

Moreover, we can find $u(x, t)$ satisfying (i)-(iii) and $\left\|u^{*}\right\|_{L^{p}\left(S_{T}\right)} \leqslant c\|f\|_{L^{p}\left(S_{T}\right)}$. 
Proof. Obviously $u(x, t)=\int_{S_{T}} f(Q, s) d \omega^{x, t}(Q, s)$ is a solution of the heat equation verifying conditions (i) and (iii) of Theorem 3.2. On the other hand Lemma 2.1 implies that the map $f \rightarrow u^{*}$ with $u$ defined as the Poisson integral of $f$ is a bounded map from $L^{p}\left(S_{T}\right) \rightarrow$ weak $-L^{p}\left(S_{T}\right)$ for $2 \leqslant p<\infty$. When $f$ is continuous and compactly supported in $S_{T}, u$ is continuous in $\bar{D}_{T}$ and

$$
\lim _{(x, t) \in D_{T} \rightarrow\left(Q_{0}, s\right)} u(x, t)=f(x, t) .
$$

Since this class of $f$ 's is dense in $L^{p}\left(S_{T}\right), 2 \leqslant p<\infty$, condition (ii) follows from a fairly standard argument.

For the uniqueness we may assume $u$ satisfies conditions (i)-(iii) with $f \equiv 0$ and, of course, we seek to prove $u \equiv 0$. We first fix a coordinate ball $B_{r_{0}}$ with center $Q_{0}$ on $\partial D$, i.e.

$$
B_{r_{0}} \cap D=\left\{x=\left(x^{\prime}, x_{n}\right):|x|<r_{0}, x_{n}>\varphi\left(x^{\prime}\right)\right\} .
$$

For $\varepsilon>0$ and sufficiently small, the function $u_{\varepsilon}(x, t)=u\left(x^{\prime}, x_{n}+\varepsilon, t\right)$ is continuous in ${\overline{\left(B_{r_{0}} \cap D\right)}}_{T}$ and since it is zero initially (condition (i))

$$
u_{\varepsilon}(x, t)=\int_{0}^{T} \int_{\partial\left(B_{r_{0}} \cap D\right)} u_{\varepsilon} d \omega_{\left(B_{r_{0}} \cap D\right)_{+}}^{x, t} \quad(t<T) .
$$

Condition (iii) and Lebesgue's dominated convergence theorem allow us to conclude that

$$
u(x, t)=\lim _{\varepsilon \rightarrow 0} u_{\varepsilon}(x, t)=\int_{0}^{T} \int_{\partial\left(B_{r_{0}} \cap D\right)} u d \omega_{\left(B_{r_{0}} \cap D\right)_{+}}^{x, t} .
$$

Since $u$ is zero on $\partial D$ we have

$$
u(x, t)=\int_{0}^{T} \int_{\partial\left(B_{r_{0}} \cap D\right) \backslash \partial D} u d \omega_{\left(B_{r_{0}} \cap D\right)_{+}}^{x, t} .
$$

It follows that, for $0 \leqslant s_{0}<T$,

$$
\lim _{(x, t) \in D_{T} \rightarrow\left(Q_{0}, s_{0}\right)} u(x, t)=0 .
$$

So $u$ is continuous in $\bar{D}_{T}$ and is zero on $\partial D \times[0, T) \cup D \times\{0\}$. Hence $u \equiv 0$ in $D_{T}$.

\section{REFERENCES}

1. R. R. Coifman and C. Fefferman, Weighted norm inequalities for maximal functions and singular integrals, Studia Math. 51 (1974), 241-250.

2. B. E. J. Dahlberg, On estimates of harmonic measure, Arch. Rational Mech. Anal. 65 (1977), $272-288$

3. On the Poisson integral for Lipschitz and $C^{1}$ domains, Studia Math. 66 (1979), 13-24. 1964.

4. A. Friedman, Partial differential equations of parabolic type, Prentice-Hall, Englewood Cliffs, N. J.,

5. J. Kemper, Temperatures in several variables: Kernel functions, representation and parabolic boundary values, Trans. Amer. Math. Soc. 107 (1972), 243-262.

6. A A boundary Harnack principle for Lipschitz domains and the principle of positive singularities, Comm. Pure Appl. Math. 25 (1972), 247-255. 
7. D. S. Jerison and C. E. Kenig, The Dirichlet problem in nonsmooth domains, Ann. of Math. (2) 113 (1981), 367-382.

8. J. Moser, A Harnack inequality for parabolic differential equations, Comm. Pure. Appl. Math. 17 (1964), 101-134.

9. B. Muckenhoupt, Weighted norm inequalities for the Hardy maximal function, Trans. Amer. Math. Soc. 165 (1972), 207-226.

10. J. M. Wu, On parabolic measures and superparabolic functions, Trans. Amer. Math. Soc. 251 (1979), 171-185.

School of Mathematics, University of Minnesota, Minneapolis, Minnesota 55455

Istituto Matematico F. Enriques, Universita di Milano, 20133 Milano, Italia 